\title{
Application of the DIVA principle to Salmonella Typhimurium vaccines in pigs avoids interference with serosurveillance programmes
}

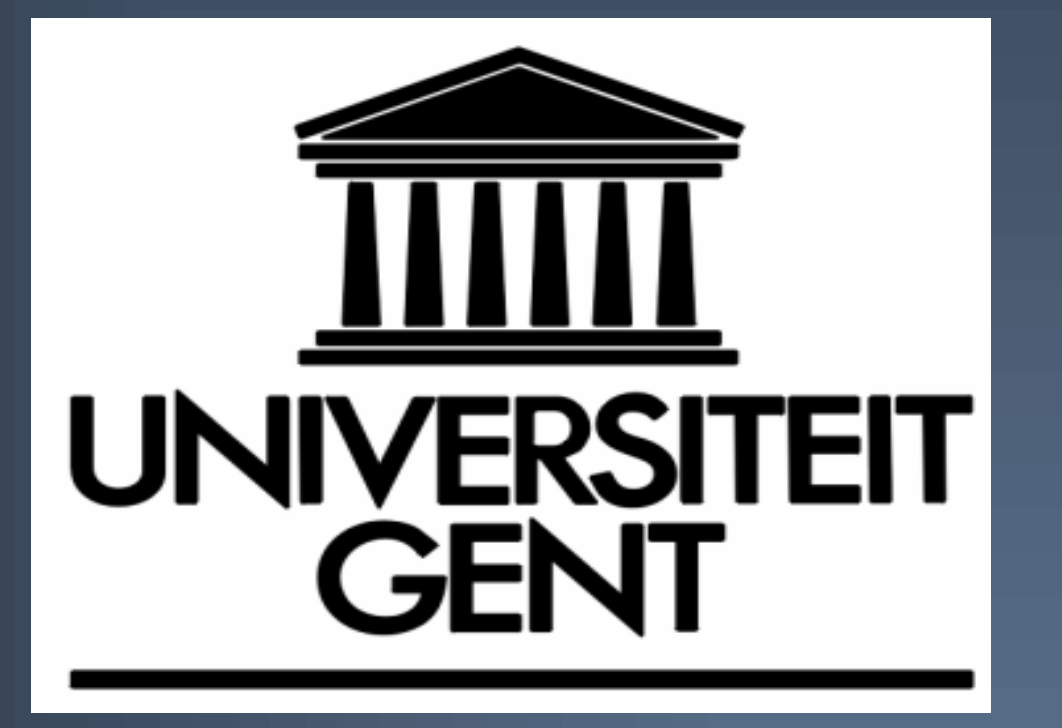

\author{
Bregje Leyman, Freddy Haesebrouck, Filip Boyen, Alexander Van Parys, Elin Verbrugghe, Frank Pasmans \\ Faculty of Veterinary Medicine, Ghent University, Department of Pathology, Bacteriology and Avian Diseases \\ Salisburylaan 133, 9820 Merelbeke, Belgium; E-mail: Bregje.Leyman@UGent.be
}

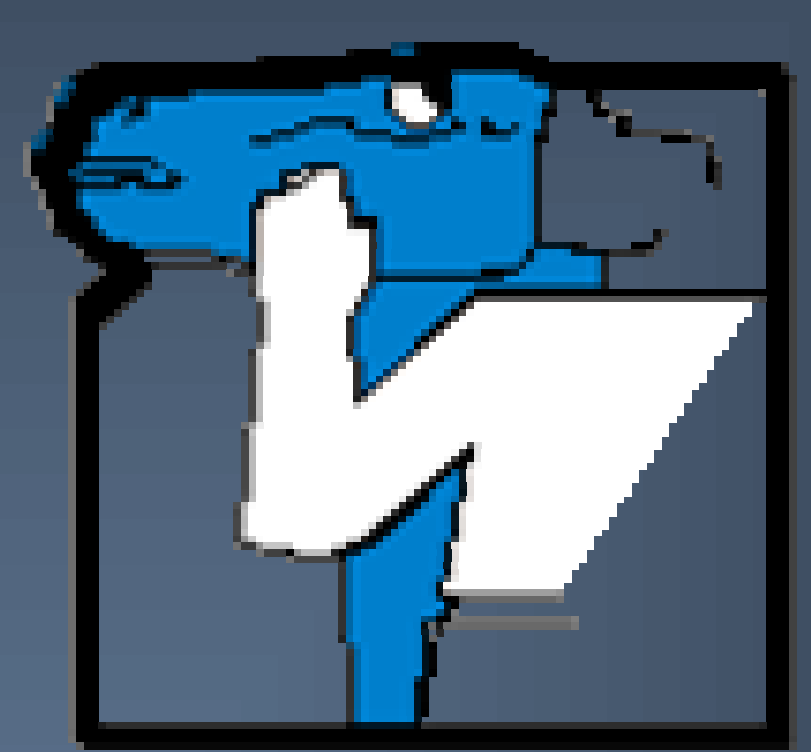

\section{Introduction}

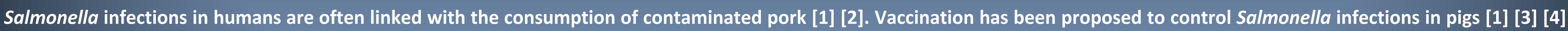

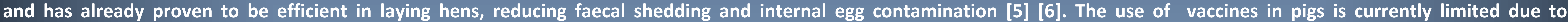

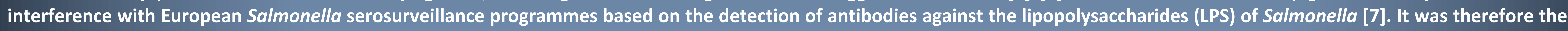

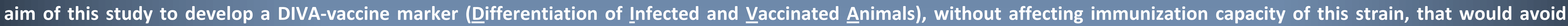
interference with current LPS-ELISA based serosurveillance programmes.

\section{Experimental objectives, methods and results}

Vaccination of mice with $\Delta r f b A, \Delta r f a L$ and $\Delta r f a J$ but not $\Delta r f a l, \Delta r f a G$ and $\Delta r f a F$ induces protection in mice against a Salmonella Typhimurium infection

We tested whether mutations in the LPS of Salmonella Typhimurium strain 112910a affect its protective capacity against a subsequent challenge with a highly virulent strain.

For that purpose, seven groups of ten mice were immunized via the orogastric route with $10^{7} \mathrm{CFU}$ of one of the LPS mutant strains (either: $\Delta r f b A, \Delta r f a L, \Delta r f a J, \Delta r f a l, \Delta r f a G$ or $\Delta r f a F$ ) or with the wild type Sallmonella Typhimurium strain 112910a. Four weeks after immunization, all mice were challenged with $10^{8} \mathrm{CFU}$ of the virulent Salmonella Typhimurium strain NCTC12023 ${ }^{\mathrm{Nal20}}$ by the orogastric route. Mice were euthanized nine days post challenge.

Conclusion:

Oral immunization of mice with Salmonella Typhimurium strain 112910a, $\Delta r f b A, \Delta r f a l$ or $\Delta r f a J$ induced a significant $(P<0.05)$ protection against subsequent challenge with NCTC12023 ${ }^{\mathrm{Nal}} 20$ in both spleen and liver compared to non immunized control animals. Results are shown in figure 1.

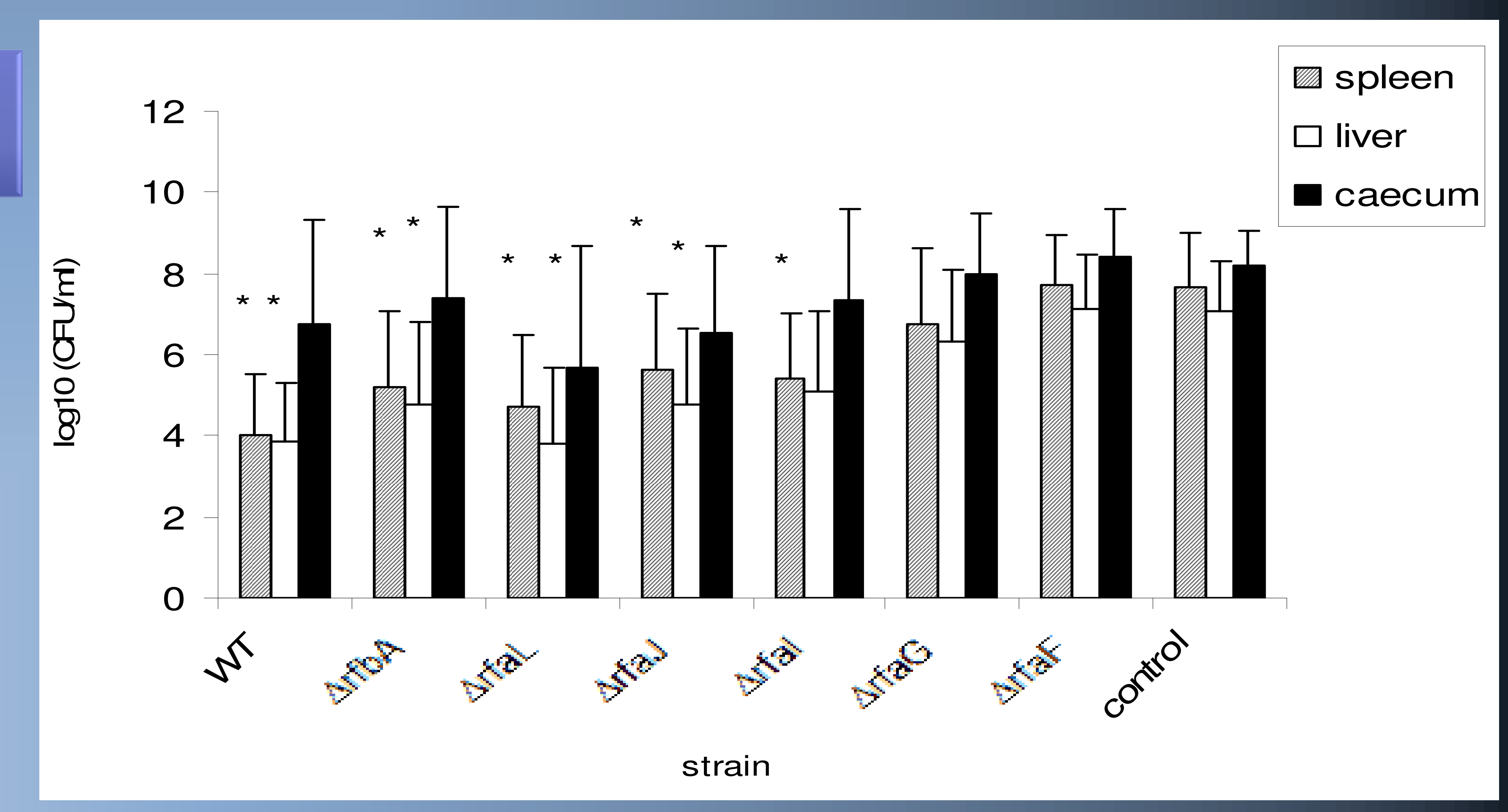

Figure 1:Recovery of Salmonella bacteria from various organs of mice immunized with either Salmonella Typhimurium, one of its isogenic LPS mutants or non immunized control animals and subsequently challenged with Salmonella Typhimurium strain NCTC12023Na|20. The mean of the log10 value of CFU per gram sample and standard deviations are shown. An asterisk refers to a significant difference with the control group $(P<0.05)$.

Pigs, immunized with the rfaJ or rfaL mutant , can be serologically differentiated from Salmonella infected animals

We examined if it was possible to discriminate between the serological response induced after vaccination of pigs with adjuvanted bacterins of either Salmonella Typhimurium strain 112910a or one of its isogenic LPS mutant strains $(\Delta r f a L ~ \Delta r f a J)$. Secondly we compared this with the serological response of pigs after infection with Salmonella Typhimurium strain 112910a.

Therefore, 14 piglets were randomly allocated to three vaccination groups ( $n=12)$ and one sham-immunized control group $(n=2)$. The animals were intramuscularly immunized with one of the formalin-inactivated Salmonella strains (either: Salmonella Typhimurium strain 112910a, $\Delta r f a J$ or $\Delta r f a L$ ) in Freund's incomplete adjuvant. To obtain sera from Salmonella Typhimurium infected piglets, one experimental group $(n=3)$ was orally inoculated with approximately $2 \times 10^{7}$ CFU of Salmonella Typhimurium strain $112910 a^{\mathrm{Nal} 20}$.

\section{Conclusion:}

Anti-Salmonella-antibody titers were detected in the serum of all immunized and infected animals, when using an in-house whole cell ELISA. No significant seroconversion was seen $(P>0.05)$ in animals immunized with inactivated $\Delta$ rfaJ or $\Delta r f a L$ strains and in sham-immuized control animals (non immunized and non infected animals), when using the commercial IDEXX ELISA. Conversely, marked seroconversion occurred in pigs immunized with the inactivated Salmonella Typhimurium strain 112910a. Results illustrate a clear differentiation between sera from piglets immunized with the $\Delta r f a J$ strain or $\Delta r f a L$ strain and sera of pigs infected with their isogenic wild type strain. Anti-Salmonella-antibody titers were detected in the serum of all immunized and infected animals, when using an in-house whole cell ELISA. Results are shown in figure 2.

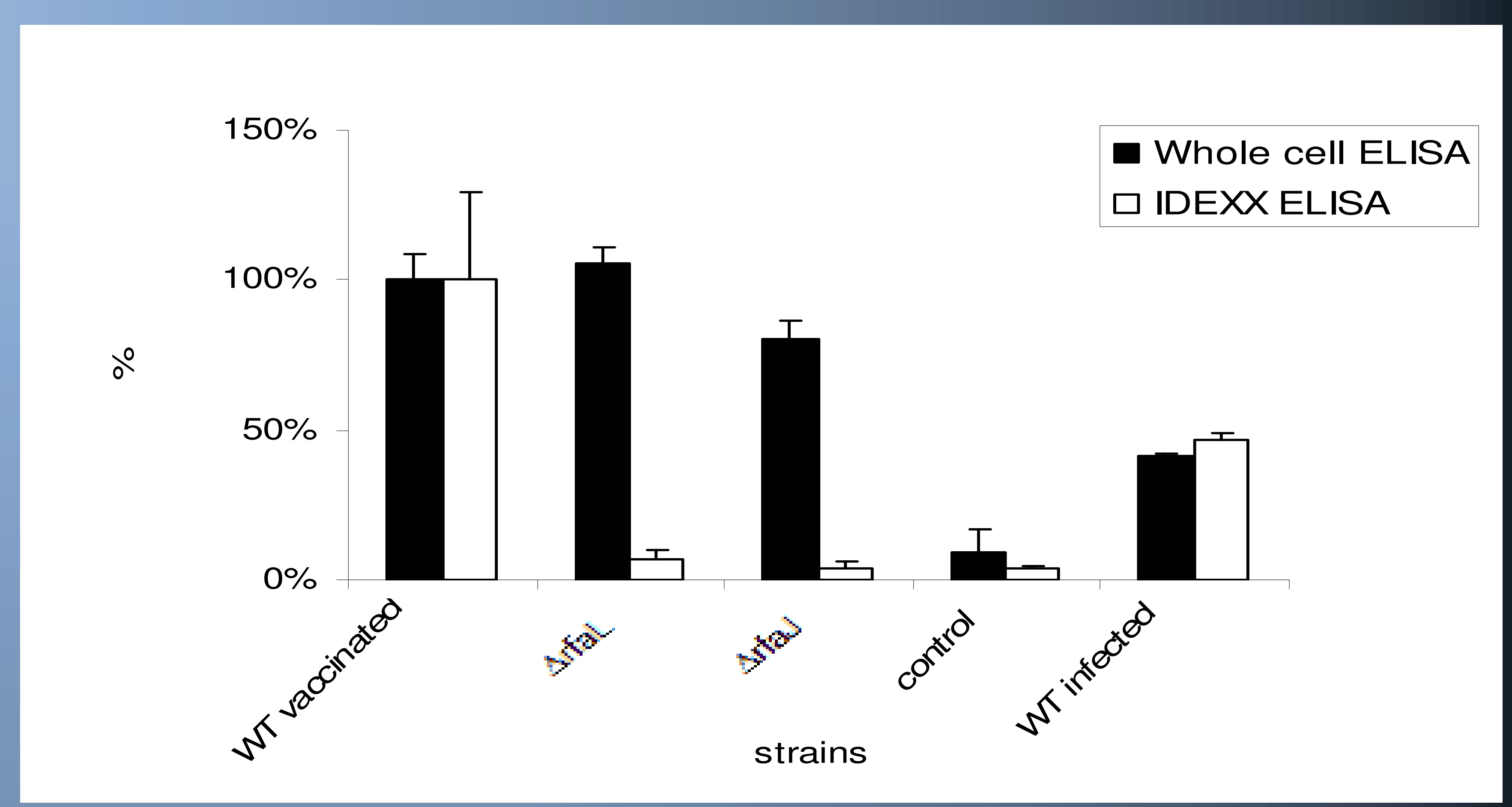

Figure 2: Serological results of pigs immunized with $\Delta r f a L, \Delta r f a J$ or Salmonella Typhimurium strain 112910a, control pigs (animals that were not immunized and not infected) and pigs infected with Salmonella Typhimurium strain 112910a Nal20. Values are represented as a percentage compared to the wild type vaccinated group.

\section{General conclusion}

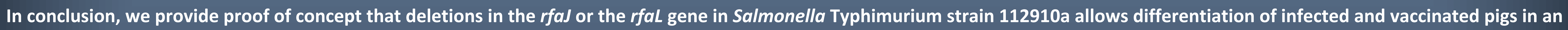
LPS based ELISA without reducing the strain's protective capacities in mice. Further research in pigs is underway.

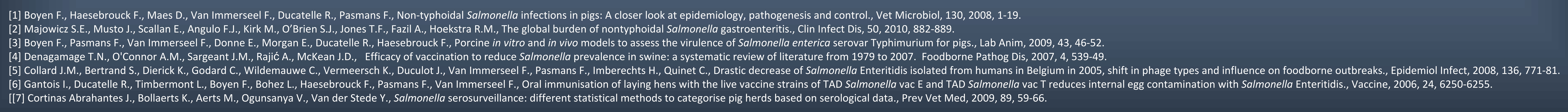

